\title{
Encontro de Anopheles (Nyssorhyncus) albitarsis s.l. (Culicidae: Anophelinae) e risco de transmissão de malária no Campus Universidade Estadual de Roraima em Boa Vista, Roraima, Brasil
}

\author{
Jaime de Liege Gama Neto ${ }^{1} \&$ Paulo Ricardo Souza Cavalcante ${ }^{2}$ \\ 1. Universidade Estadual de Roraima, Boa Vista, Museu Integrado de Roraima, Roraima, Brasil \\ 2. Universidade Estadual de Roraima, Boa Vista, Roraima, Brasil. Endereço para correspondência: Jaime de Liege \\ Gama Neto, Universidade Estadual de Roraima, Coordenação de Ciências Biológicas e da Saúde, Rua sete de \\ Setembro, $\mathrm{n}^{\circ}$ 231, Bairro: Canarinho, CEP: 69306-530 Boa Vista - Roraima - Brasil, Tel:.+55 (95) 2121-0928 E-mail: \\ jaimebio@hotmail.com
}

Recebido em : 10/01/2013. Aceito em: 18/02/2013.

\section{RESUMO}

Comunica-se o encontro de Anopheles albitarsis s.l nas dependências do Campus da Universidade Estadual de Roraima em Boa Vista/RR. An. albitarsis s.l. foi observado picando humanos nas primeiras horas da noite no peridomicílio do Campus e fêmeas foram capturadas com isca humana no período de março a junho de 2012. O encontro de An. albitarsis s.l, associado ao fluxo intenso de alunos e professores entre os campi do interior do Estado, inclusive em áreas de transmissão de malária, constitui situação de risco, sendo recomendadas ações de educação em saúde, comunicação e mobilização, bem como atividades de vigilância entomológica, visando a prevenção da transmissão da malaria no Campus da UERR em Boa Vista.

PALAVRAS-CHAVE: Insetos vetores, mosquito, savana, lavrado

\section{ABSTRACT}

We report the finding of Anopheles albitarsis s.l into the dependencies of State University of Roraima Campus in Boa Vista / RR. Anopheles albitarsis s.l was found biting humans in the early hours of the night at peridomicile and females were captured with human bait between march to june/2012. The encounter of Anopheles albitarsis s.l associated with the intense flux of students and teachers between campi rest of the state, including in malaria transmission areas, constitutes risk situation being recommended health education, communication and mobilization actions, as well as entomological surveillance activities, aimed at preventing the transmission of malaria in the UERR Boa Vista Campus.

KEY WORDS: Vectors insects, mosquitoes, savannah, lavrado.

\section{INTRODUÇÃO}

Os mosquitos anofelinos possuem importância médica pelo fato de várias das suas espécies serem vetores de parasitos do gênero Plasmodium, agente etiológico da malária humana, a mais dispersa e mais séria doença parasitária do Mundo.

Nos países tropicais e subtropicais há uma estimativa de 149 a 274 milhões de casos de malária (WHO 2011) com a severidade variando desde casos de maior ou menor gravidade em virtude da espécie do parasita inoculado e do grau de imunidade prévia do indivíduo infectado (Coura et al. 2006), até casos assintomáticos onde $\mathrm{o}$ indivíduo infectado, mesmo não apresentando sintomas, pode infectar o mosquito vetor, servindo de reservatório da doença.

Dentre as espécies vetores de malária a humanos, destacam-se aquelas incluídas no complexo de espécies Anopheles (Nyssorhyncus) albitarsis, que inclui $A n$. albitarsis Lynch-Arribalzaga, 1878, An. deaneorum Rosa-Freitas, 1989, An. marajoara Galvão e Damasceno, 1942, An. albitarsis "B"(Wilkerson et al. 1995), An. albitarsis "E"(Lehr et al. 2005) e An. albitarsis "F" (Brochero et al. 2007).

An. deaneorum é o provável vetor de malária no Estado de Rondônia (Klein et al. 1991a, b), enquanto que $A n$. marajoara parece ser o vetor primário de malária no Estado do Amapá (Conn et al. 2002) e An. albirtarsis "E" um importante vetor de malária nas regiões habitadas das savanas do município de Boa Vista, Estado de Roraima (Silva et al. 2002; Póvoa et al. 2006).

O objetivo deste trabalho é comunicar o encontro de An. albitarsis s.l. no peri e intradomicílio do Campus da Universidade Estadual de Roraima em Boa Vista, bem como chamar a atenção para a possibilidade de 
desencadeamento de um surto de malária nas dependências desse Campus.

\section{MATERIAL E MÉTODOS}

A Universidade Estadual de Roraima (UERR) possui um caráter multi-campi, com cursos de graduação distribuídos em doze dos quinze municípios que constituem o Estado de Roraima, inclusive em localidades de alta transmissão de malária, como por exemplo, nos municípios de Rorainópolis, Cantá e Caracaraí.

Esse caráter multi-campi gera um fluxo diário e constante de pessoas (professores, técnicos e alunos) que se deslocam dos campi do interior do Estado para o campus da UERR em Boa Vista e vice-versa, tanto nos períodos matutino e vespertino, quanto no noturno.

O Campus da UERR em Boa Vista está localizado em uma ecorregião dominada pela savana (lavrado roraimense) e limitada pelo rio Branco, em terrenos de baixas altitudes (aproximadamente $150 \mathrm{~m}$ ), com registro de ocorrência de An. darlingi, An. albitarsis s.l, An. braziliensis e An. Nunestovaris (RosaFreitas et al. 2007).

Recentemente, foi observada a presença de mosquitos anofelinos no peridomicílio e no interior das salas de aula desse Campus, com uma quantidade considerada de anofelinos picando humanos nas primeiras horas da noite no peridomicílio. Em março de 2012, por precaução, foi realizado a borrifação das instalações com inseticida piretróide e iniciado o monitoramento da população de anofelinos no peridomicílio do Campus.

O monitoramento foi realizado através de coletas com a utilização de isca humana (representada pelos autores deste trabalho) nos meses de março, abril, maio e junho de 2012, no intervalo de tempo compreendido entre as 18:00 e 22:00 horas, horário nos quais há um intenso fluxo de alunos, professores e técnicos no Campus.

\section{RESULTADOS E DISCUSSÃO}

Durante o período de coletas foram capturadas 17 fêmeas de anófeles, pertencentes ao complexo de espécies Anopheles (Nyssorhyncus) albitarsis, que a partir deste ponto, por não terem sido identificadas ao nível de espécie, serão referidas simplesmente como Anopheles albitarsis.

Apesar da quantidade reduzida de espécimes capturados, os dados sugerem que no campus da UERR em Boa Vista An. albitarsis tem atividade de hematofagia no intervalo de tempo compreendido entre as 18:00 e as 21:00, deixando de ocorrer a partir deste último horário, corroborando os resultados obtidos anteriormente no jardim das copaíbas, um assentamento rural localizado a $5 \mathrm{Km}$ da capital Boa Vista (Póvoa et al. 2006; Barros \& Honório 2007).

A quantidade reduzida de fêmeas coletadas pode ser consequência da borrifação realizada nas instalações do campus (Santos et al. 2007) e também do próprio padrão de abundância de An albitarsis que, em áreas de savana do município de Boa Vista, demonstra ser mais abundante no mês de junho (Póvoa et al. 2006), mês em encerramos este monitoramento.

É importante ressaltar que a existência de casos assintomáticos de malária na região amazônica (Suares et al. 2004; Alves et al. 2005), associada à presença de An. albitarsis e ao fluxo diário de humanos entre os campi dos municípios com transmissão de malária e o campus de Boa Vista, constitui fator de risco para transmissão de malária no campus da UERR em Boa Vista (Tauil et al. 1985).

Dessa forma, recomenda-se a manutenção das ações de monitoramento e controle entomológico nas dependências do campus, bem como a implantação de ações de educação em saúde, comunicação e mobilização social tanto no campus de Boa Vista quanto nos campi da UERR localizados no interior do Estado.

Adicionalmente recomenda-se a realização de estudos mais aprofundados que determinem a densidade populacional de An. albitarsis no Campus da UERR em Boa Vista, bem como a fração dessa população que sobrevive tempo suficiente para ultrapassar o ciclo extrínseco do parasito e causar infecções humanas (Santos \& Foratini 1999), visando inferir sobre o risco real de transmissão de malária e direcionar ações efetivas de prevenção da doença nesse Campus universitário.

\section{REFERÊNCIAS BIBLIOGRÁFICAS}

Alves, F.P.; Gil, L.H.S.; Marrelli, M.T.; Ribolla, P.E.M.; Camargo, E.P. \& Silva L.H.P. 2005. Assymptomatic carriers of Plasmodium spp. as infection source for malaria vector mosquitoes in the Brazilian Amazon. Journal of Medical Entomology, 42: 777-779.

Barros, F.S.M. \& Honório, N.A. 2007. Man biting rate 
seasonal variation of malaria vectors in Roraima, Brazil. Memórias do Instituto Oswaldo Cruz, 102(3): 299-302.

Brochero, H.H.L.; Li, C. \& Wilkerson, R.C. 2007. A newly recognized species in the Anopheles (Nyssorhynchus) albitarsis complex (Diptera: Culicidae) from Puerto Carreño, Colombia. American Journal of Tropical Medicine and Hygiene, 76: 1113-1117.

Conn, J.E.; Wilkerson, R.C.; Segura, N.O.; Souza, R.T.L.; Schlichting, C.D.; Wirtz, R.A. \& Póvoa, M.M. 2002. Emergence of a new neotropical malaria vector facilitated by human migration and changes in land use. American Journal of Tropical Medicine and Hygiene, 66: 18-22.

Coura, J.R.; Suárez-Mutis, M. \& Ladeia-Andrade, S. 2006. A new challenge for malaria control in Brazil: asymptomatic Plasmodium infection - A Review. Memórias do Instituto Oswaldo Cruz, 101(3): 229237.

Klein, T.A.; Lima, J.B. \& Tada, M.S. 1991 a. Comparative susceptibility of anopheline mosquitoes to Plasmodium falciparum in Rondonia, Brazil. American Journal of Tropical Medicine and Hygiene, 44: 598-603.

Klein, T.A.; Lima, J.B.; Tada, M.S. \& Miller, R. 1991 b. Comparative susceptibility of anopheline mosquitoes in Rondônia, Brazil to infection by Plasmodium vivax. American Journal of Tropical Medicine and Hygiene, 45: 463-470.

Lehr, M.A.; Kilpatrick, C.W.; Wilkerson, R.C. \& Conn, J.E. 2005. Cryptic species in the Anopheles (Nyssorhynchus) albitarsis (Diptera: Culicidae) complex: incongruence between random amplified polymorphic DNA-polymerase chain reaction identification and analysis of mitochondrial DNA COI gene sequences. Annals of the Entomological Society of America. 2005; 98: 908-917.

Póvoa, M.M.; Souza, R.T.L.; Lacerda, R.N.L.; Rosa, E.S.; Galiza, D.; Souza, J.R.; Wirtz, R.A.; Schilichting, C.D. \& Conn, J.E. 2006. The importance of Anopheles albitarsis E and An. darlingi in human malaria transmission in Boa Vista, state of Roraima, Brazil. Memórias do Instituto Oswaldo Cruz, 101(2): 163-168.

Rosa-Freitas, M.G.; Tsouris, P.A.; Peterson, T.; Honório, N.A.; Barros, F.S.M.; Aguiar, D.B.; Gurgel, H.C.; Arruda, M.E.; Vasconcelos, S.D. \& Luitgards-Moura JF. An ecoregional classification for the state of Roraima, Brazil. The importance of landscape in malaria biology. Memórias do Instituto Oswaldo Cruz, 102(3): 349-357.

Santos, R.C.; Fayal, I.A.S.; Aguiar, A.E.F.; Vieira, D.B.R. \& Póvoa, M.M. 2007. Avaliação do efeito residual de piretróides sobre anofelinos da Amazônia brasileira. Revista de Saúde Pública, 41(2): 276-83.

Santos, R.L.C. \& Forattini, O.P. 1999. Marcação-solturarecaptura para determinar o tamanho da população natural de Anopheles albitarsis 1.s. (Diptera: Culicidae). Revista de Saúde Pública, 33(3): 309-313. Silva-Vasconcelos, A.; Kato, M.Y.; Mourão, E.N.; Souza, R.T.; Lacerda, R.N.; Sibajev, A.; Tsouris, P.; Póvoa, M.M.; Momen, H. \& Rosa-Freitas, M.G. 2002. Biting indices, host-seeking activity and natural infection rates of anopheline species in Boa Vista, Roraima, Brazil from 1996 to 1998. Memórias do Instituto Oswaldo Cruz, 97(2): 151-161.

Suárez-Mutis, M.C.; Cuervo, P.; Fernandes, O. \& Coura, J.R. 2004. Evidência da presença de infecção assintomática por Plasmodium no médio rio Negro, Estado do Amazonas. Revista da Sociedade Brasileira de Medicina Tropical, 37(Supl. I): 268269.

Tauil, P.; Deane, L.; Sabroza, P. \& Ribeiro, C. 1985. A malária no Brasil. Cadernos de Saúde Pública, 1(1): 71-111.

WHO-World Health Organization. World Malaria Report, 2011. (www.who.int/malaria/ worldmalariareport2011/WMR2011noprofileslowres.pdf) Acesso em 25 de julho de 2012.

Wilkerson, R.C.; Parsons, T.J.; Klein, T.A.; Gaffigan, T.V.; Bergo, E. \& Consolim, J. 1995. Diagnosis by random amplified polymorphic DNA polymerase chain reaction of four cryptic species related to Anopheles (Nyssorhynchus) albitarsis (Diptera: Culicidae) from Paraguay, Argentina and Brazil. Journal of Medical Entomology, 32: 697-704. 